\title{
Better diet quality scores are associated with a lower risk of hypertension and non-fatal CVD in middle-aged Australian women over 15 years of follow-up
}

\author{
Jacklyn K Jackson ${ }^{1}$, Lesley K MacDonald-Wicks ${ }^{1,2}$, Mark A McEvoy ${ }^{3}$, Peta M Forder ${ }^{4}$, \\ Carl Holder ${ }^{5}$, Christopher Oldmeadow ${ }^{5}$, Julie E Byles ${ }^{4}$ and Amanda J Patterson 1,2,* \\ ${ }^{1}$ Faculty of Health and Medicine, School of Health Sciences, Department of Nutrition and Dietetics, University of \\ Newcastle, Callaghan, NSW 2308, Australia: ${ }^{2}$ Priority Research Centre for Physical Activity and Nutrition, University \\ of Newcastle, Callaghan, NSW 2308, Australia: ${ }^{3}$ Faculty of Health and Medicine, School of Medicine and Public \\ Health, Hunter Medical Research Institute, University of Newcastle, New Lambton, New South Wales, Australia: \\ ${ }^{4}$ Research Centre for Generational Health and Ageing, Hunter Medical Research Institute, University of Newcastle, \\ New Lambton, New South Wales, Australia: ${ }^{5}$ Clinical Research Design, Information Technology and Statistical \\ Support Unit, Hunter Medical Research Institute, University of Newcastle, New Lambton, New South Wales, Australia
}

Submitted 18 January 2019: Final revision received 27 May 2019: Accepted 24 June 2019: First published online 14 October 2019

\begin{abstract}
Objective: To explore if better diet quality scores as a measure of adherence to the Australian Dietary Guidelines (ADG) and the Mediterranean diet (MedDiet) are associated with a lower incidence of hypertension and non-fatal CVD.

Design: Prospective analysis of the 1946-1951 cohort of the Australian Longitudinal Study on Women's Health (ALSWH). The Australian Recommended Foods Score (ARFS) was calculated as an indicator of adherence to the ADG; the Mediterranean Diet Score (MDS) measured adherence to the MedDiet. Outcomes included hypertension and non-fatal CVD. Generalised estimating equations estimated OR and $95 \%$ CI across quartiles of diet quality scores.

Setting: Australia, 2001-2016.

Participants: 1946-1951 cohort of the ALSWH ( $n$ 5324), without CVD, hypertension and diabetes at baseline (2001), with complete FFQ data.

Results: There were 1342 new cases of hypertension and 629 new cases of non-fatal CVD over 15 years of follow-up. Multivariate analysis indicated that women reporting better adherence to the ARFS $(\geq 38 / 74)$ had $15 \%$ (95\% CI $1,28 \% ; P=0.05$ ) lower odds of hypertension and $46 \%$ (95\% CI 6, $66 \% ; P=0 \cdot 1)$ lower odds of non-fatal CVD. Women reporting better adherence to the MDS $(\geq 8 / 17)$ had $27 \%$ (95\% CI 15, $47 \% ; P=0.0006)$ lower odds of hypertension and $30 \%(95 \%$ CI $2,50 \% ; P=0.03)$ lower odds of non-fatal CVD.

Conclusions: Better adherence to diet quality scores is associated with lower risk of hypertension and non-fatal CVD. These results support the need for updated evidenced based on the ADG as well as public health nutrition policies in Australia.
\end{abstract}

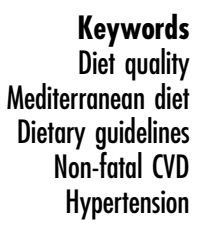

CVD represents a class of diseases that involve the heart and blood vessels, and is a major cause of death and disease burden across the globe ${ }^{(1,2)}$. In Australia, CHD (including heart attack), stroke and heart failure are the leading causes of death among Australian women ${ }^{(3)}$. Given that $40 \%$ of heart attacks in women are fatal and occur without prior warning, it is clear that CVD represents a major threat to the health of Australian women and is an issue which demands preventive action ${ }^{(3)}$.

Data published by the WHO estimate that over $75 \%$ of premature CVD is preventable ${ }^{(4)}$. This indicates that the overall burden CVD imposes on individuals, societies, governments and health-care services could be significantly reduced by addressing major CVD risk factors including dyslipidaemia, smoking, hypertension, diabetes and abdominal obesity $^{(4)}$.

Australian data suggest that high blood pressure accounts for the greatest attributable burden for CVD at $31.7 \% \%^{(5)}$. However, combined dietary risk factors, including consumption levels of fruits, vegetables, nuts, seeds, whole grains, processed meat, fibre, saturated fats, $\mathrm{Na}$, sweetened beverages and 
$n-3$ fatty acids, are thought to explain $35 \%$ of CVD burden ${ }^{(5)}$. This suggests that population interventions that target whole dietary intakes represent a powerful lifestyle strategy for preventing CVD, a concept supported by the mounting evidence demonstrating whole food and whole dietary approaches yield greater cardiovascular protective effects than those focused on single nutritive components of foods ${ }^{(6)}$.

One of the most widely studied dietary patterns linked to CVD prevention is the Mediterranean diet (MedDiet), which is characterised by high intakes of fish, olive oil, fruits, vegetables and whole grains, and a moderate intake of alcohol ${ }^{(7,8)}$. Recent literature has indicated that closer adherence to a MedDiet can reduce the risk of CVD risk factors including hypertension ${ }^{(9)}$ and high blood lipids ${ }^{(10)}$, and is associated with a lower risk of CVD incidence and mortality ${ }^{(11)}$. However, the MedDiet is a specific dietary pattern based on the eating habits of Greece, Italy and Spain in the 1940s and 1950s, and therefore may lack relevance within Australia's current food system and environment ${ }^{(12,13)}$.

Many countries have developed national dietary guidelines ${ }^{(14)}$. Dietary guidelines are evidence-based dietary recommendations promoting the types and amounts of food which should be consumed based on foods commonly available, culturally acceptable and specific to the food landscape of a country ${ }^{(15)}$. For Australians, the Australian Dietary Guidelines (ADG) have been developed with the aim of promoting health and well-being, reducing the risk of diet-related conditions and risk of chronic disease ${ }^{(15)}$. The ADG have been developed for all healthy Australians, as well as those with common diet-related CVD risk factors such as being overweight ${ }^{(15)}$.

Previous studies have indicated that, on average, middle-aged Australian women have a very low level of adherence to the MedDiet, with intakes slightly more aligned with the $\mathrm{ADG}^{(16)}$. Given the ADG were developed with the specific aim of preventing chronic disease within the Australian population, it would be interesting to explore if intakes more aligned with the ADG are associated with a similar or greater reduction in risk of hypertension and nonfatal CVD compared with the MedDiet. Therefore, the aim of the current investigation was to explore whether better diet quality scores, including the Mediterranean Diet Score (MDS; as a measure of adherence to the MedDiet) and the Australian Recommended Food Score (ARFS; as a measure of adherence to the ADG), within a representative sample of Australian women were associated with a lower incidence of self-reported hypertension and non-fatal CVD over 15 years of follow-up.

\section{Methods}

\section{The Australian Longitudinal Study on Women's Health (ALSWH)}

The ALSWH (also known as Women's Health Australia) was established in 1996 to investigate the health and well-being of Australian women. At the first survey ever conducted for this cohort in 1996, women from three age groups (1973-1978 cohort, 1946-1951 cohort, 1921-1926 cohort) were randomly selected from the Medicare database (Australia's government-funded universal health-care cover $)^{(17)}$

Women living in rural and remote areas were intentionally oversampled to allow sufficient power to analyse data by area of residence ${ }^{(18)}$. The total baseline sample recruited included over 40000 women and was found to be a nationally representative sample ${ }^{(19)}$.

Data used for the current study were derived only from the 1946-1951 cohort of the ALSWH. Survey 1 was conducted in 1996 and women in this cohort were surveyed in 1998 and every 3 years following ${ }^{(19)}$, with the most recent survey conducted in 2016. In total, data from two FFQ conducted in 2001 (as part of Survey 3) and 2013 (as part of Survey 7) were included in the current study.

Women from the 1946-1951 cohort were excluded from the analysis if they reported a diagnosis of hypertension, CVD (including heart disease, stroke or thrombosis) or diabetes at or before baseline. Women with missing FFQ data at either Survey 3 or Survey 7 were excluded. Women were also excluded if they had fewer than two follow-up surveys from baseline (Fig. 1)

The Human Research and Ethics Committee of the University of Newcastle and the University of Queensland approved the study methods. Further details on the sample and methods used by the ALSWH have been reported elsewhere and are available at www.alswh.org.au.

\section{Assessment of dietary intake}

Dietary intake was assessed using an FFQ known as the Dietary Questionnaire for Epidemiological Studies (DQES), Version $2^{(20)}$. The DQES was included as part of Survey 3 and Survey 7 only, collected in 2001 and 2013, respectively, from all participants.

The DQES asked respondents to report their usual consumption of seventy-four foods and beverages and six alcoholic beverages over the preceding 12 months, using a 10-point frequency option ranging from 'never' up to ' 3 or more times per day'. Portion size photographs were used to adjust the serving size for vegetables, meat and casseroles. Additional questions were asked about the total number of daily servings of fruit, vegetables, bread, dairy products, eggs, fat spreads and sugars, as well as asking the type of bread, dairy products and fat spread used. Both the development of the DQES and its validation in a sample of Australian women have been previously reported $^{(21)}$.

\section{Assessment of diet quality}

Two diet quality scores were calculated: the $\mathrm{ARFS}^{(22)}$ and the $\operatorname{MDS}^{(8)}$. 


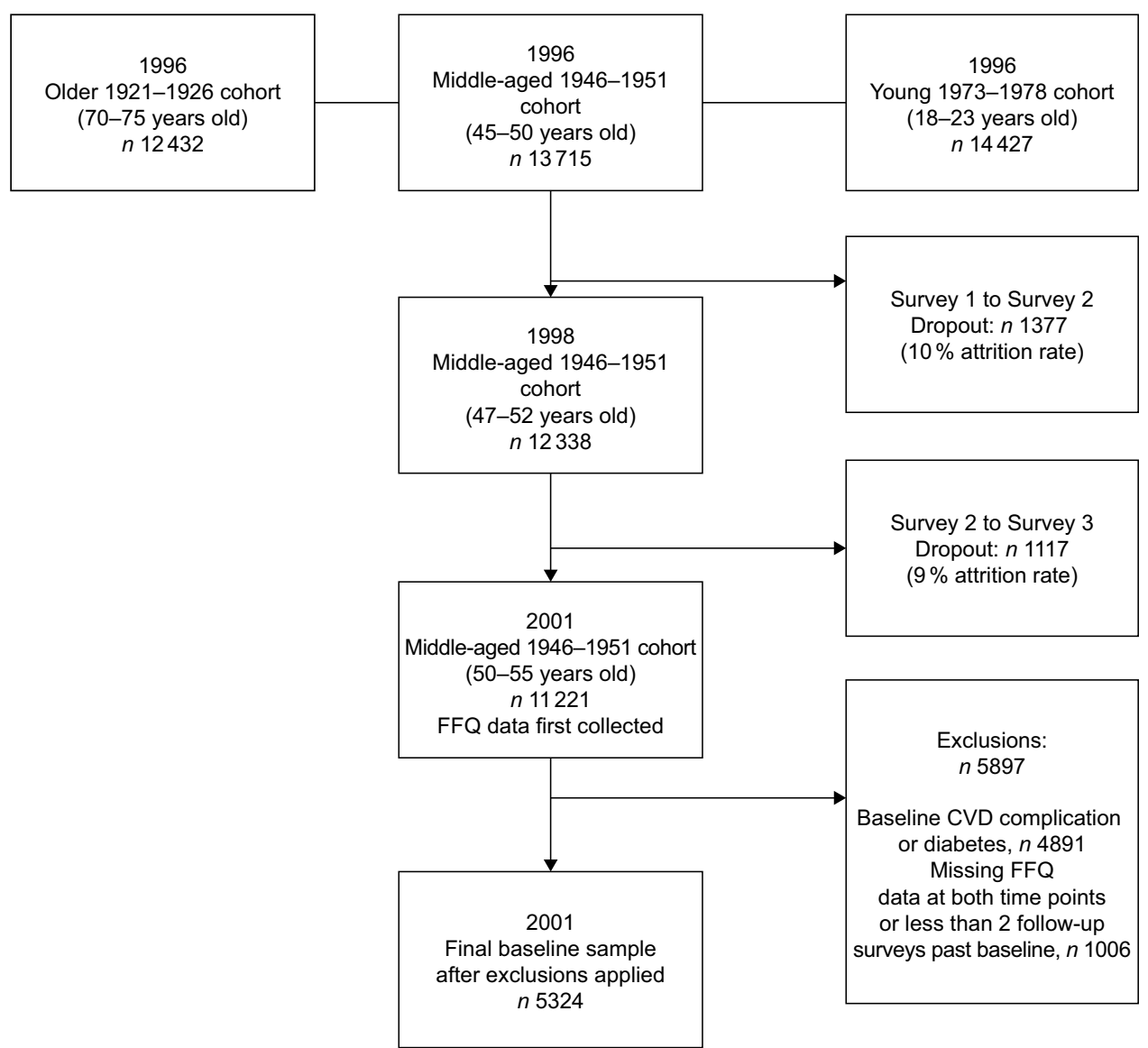

Fig. 1 Australian Longitudinal Study of Women's Health: flowchart of the 1946-1951 cohort participants

The ARFS assessed diet quality by calculating consistency with national recommendations of the dietary guidelines for Australian adults (ADG) and the core foods outlined in the Australian Guide to Healthy Eating, where higher ARFS represents greater adherence to the ADG and better diet quality ${ }^{(22)}$. For example, FFQ items including fruits and vegetables consumed $<1$ time/week were scored 0 and those consumed $\geq 1$ time/week were scored 1 . Meat FFQ items scored 0 if consumed $<1$ time/month or $>5$ times/week, and scored 1 if consumed 1-4 times/week. Additional points were also awarded for type and quality of core food intake consistent with national dietary recommendations. A maximum of 2 points were added for alcohol consumption: 1 point for moderate frequency (up to $4 \mathrm{~d}$ /week) and 1 point for moderate quantity ( 1 or 2 standard drinks, when alcohol is consumed). The maximum possible ARFS is 74, which was the total of the food group-based subscores, including vegetable score (maximum score of 22), fruit score (maximum of 14), grain score (maximum of 14), dairy score (maximum of 7 ), nuts/ beans/soya/egg score (maximum of 7), meat score (maximum of 5), fish score (maximum of 2), fat score (maximum of 1) and alcohol score (maximum of 2). A detailed explanation for calculating the ARFS is given in the online supplementary material, Supplemental Table $S 1^{(16,22)}$.

We calculated the MDS as outlined by Stefler et al. ${ }^{(8)}$, with one modification. Olive oil consumption is not collected as part of the DQES. Therefore, the MDS scoring was modified to match the information within the DQES and the MDS fat component score was awarded if women reported consuming monounsaturated fat $\operatorname{spread}^{(8)}$. The maximum MDS possible was 17; the criteria for calculating the MDS are reported in detail in the online supplementary material, Supplemental Table S2 ${ }^{(16)}$.

\section{Ascertainment of bypertension and non-fatal CVD}

Outcomes of interest included self-reported doctor diagnosis of hypertension and non-fatal CVD, reported at each survey. Cases of non-fatal CVD encompassed self-reported doctor diagnosis of heart disease, stroke or thrombosis.

Self-reported data on hypertension and non-fatal CVD were available from each survey. At Survey 1 in 1996, participants were asked if a doctor ever diagnosed them with hypertension, heart disease, stroke and/or thrombosis. For 
example, women were asked 'Have you ever been told by a doctor that you have: Heart disease?' At all subsequent surveys, participants were asked if they had been diagnosed with hypertension or non-fatal CVD by a doctor in the last 2-3 years (to coincide with previous surveys). For example, at Survey 2 women were asked 'Have you EVER been told by a doctor that you have hypertension (high blood pressure)?' to which responses included 'yes, in the last 2 years' and 'yes, more than 2 years ago'. At Survey 3 and subsequent surveys (Surveys 4, 5, 6, 7 and 8), women were asked 'In the PAST THREE YEARS, have you been diagnosed or treated for: Heart disease (including heart attack, angina), Hypertension (high blood pressure), Stroke or thrombosis (blood clot)?' Thrombosis was asked at all survey periods, except Survey 5 .

If women reported having hypertension, heart disease, stroke or thrombosis at Survey 1, 2 or 3, they were excluded from the analysis. A case of non-fatal CVD was defined as a new report of heart disease, stroke or thrombosis at Surveys 4 to 8 . A case of hypertension was defined as a new report of hypertension at Surveys 4 to 8.

Previously, the examination between self-reported hypertension and hypertension medication use in the 1946-1951 cohort indicated a high level of agreement between measures $(89 \%)^{(23)}$ and the use of self-reported stroke data was previously validated ${ }^{(24)}$.

\section{Covariates}

At every survey, participants were asked to provide information on a range of demographic and socio-economic factors and health risk behaviours. Covariates relevant to the current analysis include socio-economic status, level of education, smoking status, BMI, physical activity level, country of birth, level of alcohol intake and menopause status.

Socio-economic status was determined based on how well participants reported they could manage on the income they have available. Response options were categorized as: low ('it is impossible' or 'it is difficult all the time'); intermediate ('it is difficult some of the time' or 'it is not too bad'); or high ('it is easy') ${ }^{(25)}$. Level of education was determined as: low (if the highest level of education reported was 'no formal qualifications' or 'school or intermediate certificate or equivalent'); intermediate (if response was 'high school or leaving certificate, trade/ apprenticeship, or certificate or diploma'); or high (if level of education was classified as university degree and postgraduate degree'). Smoking status was defined as 'non-smoker', 'former smoker' or 'current smoker'.

Participants were asked to report their height and weight; from this, BMI was calculated and categorised as underweight $\left(\mathrm{BMI}<18.50 \mathrm{~kg} / \mathrm{m}^{2}\right)$, healthy weight $\left(\mathrm{BMI}=18.50-24.99 \mathrm{~kg} / \mathrm{m}^{2}\right), \quad$ overweight $\quad(\mathrm{BMI}=25.00$ $\left.29.99 \mathrm{~kg} / \mathrm{m}^{2}\right)$ or obese $\left(\mathrm{BMI} \geq 30.00 \mathrm{~kg} / \mathrm{m}^{2}\right)^{(26)}$. Selfreported BMI data from the ALSWH were previously validated $^{(27)}$. Physical activity levels were derived from validated questions on the frequency and duration of walking (for recreation or transport) and from moderateand vigorous-intensity activity in the last week. From this, physical activity level was defined as 'sedentary/low' $(<600 \mathrm{MET} \times \mathrm{min} /$ week $)$ or 'moderate/high' $\quad \geq 600$ MET $\times$ min/week $)^{(28)}$, where MET is metabolic equivalents of task. At Survey 1, participants were asked to report their country of birth, from which responses were categorised as 'Australian born', 'other English-speaking background', 'Europe', 'Asia' and 'Other'. Alcohol status was also derived and categorised as per the National Health and Medical Research Council's alcohol guidelines. Categories included 'non-drinker', 'rarely drinks', 'low-risk drinker' ( $\leq 2$ standard drinks/d), 'risky drinker' (3-4 standard drinks/d) and 'high-risk drinker' ( $\geq 5$ standard drinks/d) ${ }^{(29)}$. Menopause status was determined using questions on hysterectomy, oophorectomy, hormone therapy and menstrual patterns, and categorised as 'hysterectomy and/or oophorectomy', 'hormone therapy use', 'premenopausal', 'perimenopausal' or 'postmenopausal'(30).

\section{Covariate selection}

Using the software program DAGitty ${ }^{(31)}$, a directed acyclic graph was used to explore the association between the exposure and outcome variables, based on the literature indicating multiple potential confounding variables. From the causal diagram shown in the online supplementary material, Supplemental Fig. S1, various potential confounding variables were identified. These included race, socio-economic status, education and physical activity. Therefore, in addition to our unadjusted model, we present two adjusted models: model 1 is adjusted for energy, age, race (birth country), socio-economic status and education; and model 2 is adjusted for model 1 variables as well as physical activity level, BMI and smoking. Alcohol was also identified as a potential factor confounding the relationship between diet quality and CVD; however, was not adjusted for in our models as it is already accounted for within both diet quality scores.

\begin{abstract}
Analysis
We analysed the prospective association between diet quality scores including the ARFS and MDS (based on the FFQ data collected at Survey 3 and updated at Survey 7) and incidence of hypertension and non-fatal CVD (Surveys 4-8), using generalised estimating equations analysis. An independent correlation structure was used for the generalised estimating equations model, due to the 12-year gap between individual diet quality measures. This structure was supported by an improved model fit, as evidenced by a lower value of the quasi-likelihood under independence model criterion. Each participant contributed only one endpoint and the cohort at risk of each 3-year follow-up period included only those who had not yet reported
\end{abstract}


hypertension or a non-fatal CVD event at the beginning of each follow-up period.

For analyses, participants were divided into quartiles (Q1-Q4) based on their diet quality scores (ARFS and MDS, separately). The lowest quartile (Q1) for diet quality scores represents the reference category.

Generalised estimating equations models with timevarying covariates were used for all analyses using the statistical software package Stata version 14.2, to estimate OR and corresponding $95 \%$ CI. Statistical significance was defined as $P<0.05$.

\section{Results}

\section{Characteristics of study population}

A total of 5324 women were included at baseline (2001; Fig. 1), with a mean age of 52.4 (SD 1.5) years. Women in the highest quartile for diet quality scores (ARFS or MDS) were significantly more likely to be highly educated (university bachelor's degree) and to use a multivitamin or other supplement. Women reporting higher diet quality scores were also less likely to smoke cigarettes, less likely to partake in risky or high-risk alcoholic drinking behaviours, and less likely to be physically inactive (Table 1).

\section{Dietary intakes based on diet quality scores}

The median ARFS for our sample was 32 (interquartile range 12) out of a maximum possible score of 74 . In addition, the median MDS for our sample was 7 (interquartile range 2) out of a maximum possible score of 17 . Women reporting higher diet quality scores were on average more likely to consume greater servings of fruits, vegetables and grains each day. Higher MDS was associated with significantly lower average intakes of processed meats (servings/d) and meat (servings/d), but slightly higher average intakes of discretionary choices (servings/d), including snack foods and confectionery. Women reporting higher ARFS were significantly more likely to consume on average more meat (servings/d) and dairy (servings/d; Table 2).

\section{Incidence of bypertension}

During 15 years of follow-up, 1552 (29\%) of the 5324 participants reported having a diagnosis of hypertension. The relationship between quartiles of diet quality scores and hypertension is reported in Table 3. Women reporting a higher ARFS (Q4, ARFS $\geq 38)$ were at a $15 \%(O R=0.85$, $95 \%$ CI 0.72, 0.99; $\left.P_{\text {trend }}=0.05\right)$ lower risk of hypertension, compared with women reporting ARFS in the lowest quartile (Q1, ARFS $\leq 25)$ in our multivariate model (model 2). Women reporting a higher MDS (Q4, MDS $\geq 8$ ) were at a $27 \%\left(\mathrm{OR}=0.73,95 \%\right.$ CI 0.63, 0.85; $\left.P_{\text {trend }}=0.0006\right)$ lower risk of hypertension compared with women reporting MDS in the lowest quartile (Q1, MDS $\leq 5)$ in our multivariate model (model 2).

\section{Incidence of non-fatal CVD}

During 15 years of follow-up, 629 (12\%) participants reported having a diagnosis of non-fatal CVD. Of this sample, approximately $8.5 \%$ reported a diagnosis of heart disease, $3 \%$ reported a diagnosis of thrombosis and $2 \%$ reported a diagnosis of stroke.

The relationship between quartiles of diet quality scores and non-fatal CVD is reported in Table 4 . Women reporting a higher ARFS (Q4, ARFS $\geq 38$ ) were at a $46 \%(\mathrm{OR}=0 \cdot 64$, $95 \%$ CI 0.44, 0.94; $\left.P_{\text {trend }}=0 \cdot 1\right)$ lower risk of non-fatal CVD, compared with women reporting ARFS in the lowest quartile (Q1, ARFS $\leq 25)$ in our multivariate model (model 2). Women reporting a higher MDS (Q4, MDS $\geq 8$ ) were at a $30 \%\left(\mathrm{OR}=0.70,95 \% \mathrm{CI} 0.50,0.98 ; P_{\text {trend }}<0.03\right)$ lower risk of non-fatal CVD compared with women reporting MDS in the lowest quartile (Q1, MDS $\leq 5$ ) in our multivariate model (model 2).

\section{Discussion}

Within our sample of middle-aged Australian women, statistically significant inverse associations between diet quality scores and incidence of hypertension and non-fatal CVD were found over 15 years of follow-up. In particular, we observed greater risk reduction in hypertension with increasing adherence to the MDS compared with the ARFS. However, we also observed a very large risk reduction in non-fatal CVD with increased adherence to the ARFS compared with the MDS.

The MedDiet has been one of the most studied dietary patterns in recent years and has been consistently recognised for exerting potent cardiovascular protective effects, which have been attributed to the sum of its components ${ }^{(32)}$. Although it has been widely argued that the MedDiet does not represent one consistent dietary pattern, it is frequently defined as a plant-based diet, high in cereals, fruits, vegetables, legumes and nuts ${ }^{(33)}$. The MedDiet will generally include small amounts of meat and dairy products, olive oil as the main source of fat, and a moderate intake of alcohol ${ }^{(33)}$. Many of these dietary components are also promoted within the ADG; however, lean red meats and poultry have been grouped with fish, tofu, nuts, seeds and legumes (meat alternatives) as a class of foods which should be consumed in quantities of 1-3 servings $/ \mathrm{d}^{(15)}$. Daily consumption of dairy foods including milk, yoghurt, cheese and/or alternatives is also recommended, and unspecified unsaturated fat (polyunsaturated and monounsaturated) spreads and oils are recommended in small amounts ${ }^{(15)}$. These general concepts have been captured in our baseline diet data, as we see that higher ARFS and MDS were associated with higher average intakes 


\section{Public Health Nutrition}

Table 1 Baseline characteristics of all study participants ( $n$ 5324) by quartiles of diet quality scores; $1946-1951$ cohort of the Australian Longitudinal Study of Women's Health

\begin{tabular}{|c|c|c|c|c|c|c|c|c|c|c|}
\hline & \multicolumn{5}{|c|}{ ARFS (maximum score $=74$ ) } & \multicolumn{5}{|c|}{ MDS $($ maximum score $=17$ ) } \\
\hline & $\begin{array}{c}\text { Q1 } \\
(A R F S \leq 25)\end{array}$ & $\begin{array}{c}\mathrm{Q} 2 \\
(\mathrm{ARFS}=26-31)\end{array}$ & $\begin{array}{c}\text { Q3 } \\
(\text { ARFS }=32-37)\end{array}$ & $\begin{array}{c}\mathrm{Q} 4 \\
(\mathrm{ARFS} \geq 38)\end{array}$ & $P$ value & $\begin{array}{c}\mathrm{Q1} \\
(\mathrm{MDS} \leq 5)\end{array}$ & $\begin{array}{c}\text { Q2 } \\
(\mathrm{MDS}=6)\end{array}$ & $\begin{array}{c}\text { Q3 } \\
(\mathrm{MDS}=7)\end{array}$ & $\begin{array}{c}\text { Q4 } \\
(\mathrm{MDS} \geq 8)\end{array}$ & $P$ value \\
\hline Women $(n)$ & 1288 & 1300 & 1394 & 1342 & - & 1286 & 1015 & 1185 & 1838 & - \\
\hline Age (years) & & & & & 0.2 & & & & & 0.03 \\
\hline Mean & 52.4 & 52.4 & 52.5 & 52.5 & & $52 \cdot 3$ & 52.4 & 52.5 & 52.5 & \\
\hline SD & 1.5 & 1.5 & 1.5 & 1.4 & & 1.5 & 1.4 & 1.5 & 1.4 & \\
\hline BMI $\left(\mathrm{kg} / \mathrm{m}^{2}\right)$ & & & & & 0.1 & & & & & $0 \cdot 1$ \\
\hline Median & $25 \cdot 0$ & 24.8 & 24.8 & 24.8 & & 24.8 & 24.8 & $25 \cdot 0$ & 24.8 & \\
\hline IQR & $6 \cdot 2$ & 5.9 & $5 \cdot 1$ & $5 \cdot 1$ & & $5 \cdot 8$ & 5.4 & 5.5 & 5.6 & \\
\hline Australian born (\%) & $76 \cdot 2$ & $78 \cdot 2$ & $77 \cdot 0$ & $76 \cdot 6$ & 0.7 & $78 \cdot 6$ & $79 \cdot 4$ & $76 \cdot 3$ & $74 \cdot 3$ & 0.1 \\
\hline Urban area of residence (\%) & $36 \cdot 0$ & 35.7 & $36 \cdot 6$ & $35 \cdot 2$ & 0.9 & $36 \cdot 4$ & 34.0 & 35.9 & $36 \cdot 6$ & 0.007 \\
\hline Highly educated (\%) & $13 \cdot 2$ & $18 \cdot 6$ & $20 \cdot 8$ & $19 \cdot 0$ & $<0.0001$ & $15 \cdot 7$ & 17.5 & $16 \cdot 0$ & $21 \cdot 0$ & $<0.0001$ \\
\hline Overweight or obese (\%) & $49 \cdot 3$ & 47.9 & $47 \cdot 7$ & $46 \cdot 2$ & $<0.0001$ & $47 \cdot 1$ & 47.5 & $49 \cdot 4$ & $47 \cdot 2$ & 0.1 \\
\hline \multicolumn{11}{|l|}{ Smoking (\%) } \\
\hline Current & $19 \cdot 4$ & 11.8 & 11.6 & 9.7 & $<0.0001$ & 21.5 & $15 \cdot 5$ & $11 \cdot 2$ & $6 \cdot 9$ & $<0.0001$ \\
\hline Former & 21.8 & 25.5 & 26.5 & $24 \cdot 2$ & & $27 \cdot 8$ & $23 \cdot 6$ & $23 \cdot \overline{6}$ & 23.1 & \\
\hline Risky/high-risk alcohol drinker (\%) & 6.2 & 5.5 & 5.5 & 3.6 & $<0.0001$ & 13.7 & 4.0 & 3.3 & 1.1 & $<0.0001$ \\
\hline Physically inactive (\%) & $63 \cdot 3$ & $55 \cdot 0$ & 49.5 & $40 \cdot 1$ & $<0.0001$ & $56 \cdot 4$ & $53 \cdot 7$ & 51.9 & $47 \cdot 4$ & $<0.0001$ \\
\hline Postmenopausal (\%) & $25 \cdot 1$ & $25 \cdot 0$ & 25.4 & $26 \cdot 0$ & 0.5 & 25.5 & $25 \cdot 6$ & $23 \cdot 7$ & $26 \cdot 2$ & 0.1 \\
\hline Hormone replacement therapy use (\%) & 31.9 & $32 \cdot 2$ & 30.6 & $30 \cdot 0$ & 0.6 & 33.5 & 32.8 & $31 \cdot 2$ & 28.6 & 0.02 \\
\hline Using multivitamin/supplement & 49.6 & $57 \cdot 1$ & $60 \cdot 3$ & $64 \cdot 2$ & $<0.0001$ & $54 \cdot 1$ & 54.9 & 60.5 & 60.5 & $<0.0001$ \\
\hline
\end{tabular}

ARFS, Australian Recommended Food Score; MDS, Mediterranean Diet Score; Q, quartile; IQR, interquartile range.

Total baseline sample, $n$ 5324. Quartiles are based on ARFS or MDS separately. Age presented as mean and standard deviation; BMI presented as median and IQR. Incomplete observations for: BMI ( $n 5039)$, country of birth ( $n 5278)$, smoking status ( $n$ 5312), area of residence ( $n$ 5303), level of education ( $n 5290)$ and (National Health and Medical Research Council) alcohol status ( $n$ 5038). P value represents level of significance for difference found through ANOVA for continuous variables and $\chi^{2}$ tests for categorical variables. 
Table 2 Summary of dietary intakes of all study participants $(n$ 5324) by quartiles of diet quality scores; $1946-1951$ cohort of the Australian Longitudinal Study of Women's Health

\begin{tabular}{|c|c|c|c|c|c|c|c|c|c|c|c|c|c|c|c|c|c|c|}
\hline & \multicolumn{9}{|c|}{ ARFS $($ maximum score $=74$ ) } & \multicolumn{9}{|c|}{ MDS (maximum score $=17$ ) } \\
\hline & \multicolumn{2}{|c|}{$\begin{array}{c}\text { Q1 } \\
(\text { ARFS } \leq 25) \\
\end{array}$} & \multicolumn{2}{|c|}{$\begin{array}{c}\mathrm{Q} 2 \\
(\mathrm{ARFS}=26-31) \\
\end{array}$} & \multicolumn{2}{|c|}{$\begin{array}{c}\text { Q3 } \\
(\text { ARFS }=32-37) \\
\end{array}$} & \multicolumn{2}{|c|}{$\begin{array}{c}\mathrm{Q} 4 \\
(\mathrm{ARFS} \geq 38)\end{array}$} & \multirow[b]{2}{*}{$P$ value } & \multicolumn{2}{|c|}{$\begin{array}{c}\mathrm{Q} 1 \\
(\mathrm{MDS} \leq 5)\end{array}$} & \multicolumn{2}{|c|}{$\begin{array}{c}\mathrm{Q} 2 \\
(\mathrm{MDS}=6) \\
\end{array}$} & \multicolumn{2}{|c|}{$\begin{array}{c}\mathrm{Q3} \\
(\mathrm{MDS}=7) \\
\end{array}$} & \multicolumn{2}{|c|}{$\begin{array}{c}\mathrm{Q} 4 \\
(\mathrm{MDS} \geq 8) \\
\end{array}$} & \multirow[b]{2}{*}{$P$ value } \\
\hline & Median & IQR & Median & IQR & Median & IQR & Median & IQR & & Median & IQR & Median & IQR & Median & IQR & Median & IQR & \\
\hline ARFS & 21 & 6 & 29 & 3 & 34 & 3 & 41 & 6 & $<0.0001$ & 27 & 12 & 31 & 11 & 33 & 11 & 35 & 10 & $<0.0001$ \\
\hline MDS & 6 & 2 & 7 & 3 & 7 & 2 & 8 & 1 & $<0.0001$ & 5 & 1 & 6 & 0 & 7 & 0 & 8 & 1 & $<0.0001$ \\
\hline Vegetable (servings/d) & 1.8 & 1.4 & $2 \cdot 0$ & $1 \cdot 2$ & $2 \cdot 1$ & $1 \cdot 2$ & $2 \cdot 3$ & $1 \cdot 2$ & $<0.0001$ & $1 \cdot 8$ & $1 \cdot 3$ & 1.9 & $1 \cdot 1$ & $2 \cdot 1$ & $1 \cdot 2$ & $2 \cdot 3$ & 1.4 & $<0.0001$ \\
\hline Fruit (servings/d) & 1.0 & $1 \cdot 2$ & $1 \cdot 7$ & 1.4 & $2 \cdot 0$ & 1.4 & $2 \cdot 4$ & 1.4 & $<0.0001$ & 0.9 & 1.0 & 1.5 & 1.2 & 1.9 & 1.3 & 2.5 & 1.3 & $<0.0001$ \\
\hline Grains (servings/d) & $2 \cdot 9$ & 1.9 & 3.4 & 1.9 & 3.9 & $2 \cdot 0$ & $4 \cdot 2$ & $2 \cdot 2$ & $<0.0001$ & $2 \cdot 6$ & $1 \cdot 7$ & $3 \cdot 2$ & 1.9 & $3 \cdot 6$ & 1.9 & $4 \cdot 2$ & $2 \cdot 1$ & $<0.0001$ \\
\hline Dairy (servings/d) & 1.5 & 0.9 & 1.6 & 0.9 & $1 \cdot 7$ & 1.0 & 1.9 & $1 \cdot 0$ & $<0.0001$ & 1.7 & 0.9 & 1.7 & 1.0 & 1.7 & $1 \cdot 0$ & 1.6 & $1 \cdot 1$ & 0.2 \\
\hline $\begin{array}{l}\text { Processed meat } \\
\text { (servings/d) }\end{array}$ & 0.2 & 0.3 & $0 \cdot 2$ & 0.3 & 0.2 & 0.3 & 0.2 & 0.3 & 0.45 & 0.2 & 0.3 & 0.2 & 0.3 & 0.2 & 0.3 & $0 \cdot 1$ & 0.3 & $<0.0001$ \\
\hline Meat (servings/d) & 1.4 & 1.2 & 1.6 & $1 \cdot 2$ & $1 \cdot 7$ & $1 \cdot 2$ & 1.9 & $1 \cdot 2$ & $<0.0001$ & 1.9 & 1.3 & 1.6 & 1.3 & 1.7 & 1.2 & 1.6 & 1.0 & $<0.0001$ \\
\hline $\begin{array}{l}\text { Discretionary choices } \\
\text { (servings/d) }\end{array}$ & $1 \cdot 6$ & $1 . \overline{5}$ & $1 \cdot 6$ & 1.4 & $1 \cdot 7$ & 1.5 & $1 \cdot 7$ & $1 . \overline{5}$ & 0.6 & 1.5 & 1.5 & $1 \cdot 6$ & 1.5 & $1 \cdot 7$ & $1 . \overline{5}$ & $1 \cdot 7$ & 1.5 & 0.01 \\
\hline Energy $(\mathrm{kJ} / \mathrm{d})$ & 5561 & 2615 & 6089 & 2495 & 6421 & 2568 & 6908 & 2683 & $<0.0001$ & 5656 & 2617 & 5937 & 2527 & 6303 & 2586 & 6761 & 2668 & $<0.0001$ \\
\hline Total fat $(\mathrm{g} / \mathrm{d})$ & 55 & 30 & 57 & 32 & 59 & 31 & 61 & 30 & $<0.0001$ & 58 & 32 & 57 & 31 & 58 & 31 & 60 & 31 & 0.001 \\
\hline $\begin{array}{l}\text { Total energy } \\
\text { contribution from } \\
\text { fat }(\%)\end{array}$ & 38 & 8 & 36 & 8 & 35 & 8 & 34 & 7 & $<0.0001$ & 38 & 7 & 36 & 8 & 35 & 8 & 33 & 8 & $<0.0001$ \\
\hline Saturated fat $(g / d)$ & 22 & 14 & 22 & 14 & 22 & 14 & 23 & 13 & 0.43 & 24 & 14 & 23 & 14 & 23 & 14 & 22 & 14 & 0.02 \\
\hline $\begin{array}{l}\text { Monounsaturated } \\
\text { fat }(g / d)\end{array}$ & 19 & 11 & 20 & 11 & 21 & 11 & 22 & 11 & $<0.0001$ & 20 & 12 & 20 & 11 & 20 & 11 & 21 & 11 & 0.0002 \\
\hline $\begin{array}{l}\text { Polyunsaturated } \\
\text { fat }(\mathrm{g} / \mathrm{d})\end{array}$ & 8 & 6 & 8 & 6 & 9 & 6 & 10 & 7 & $<0.0001$ & 8 & 6 & 8 & 6 & 9 & 6 & 10 & 7 & $<0.0001$ \\
\hline $\begin{array}{l}\text { Dietary cholesterol } \\
(\mathrm{mg} / \mathrm{d})\end{array}$ & 203 & 123 & 213 & 116 & 221 & 118 & 236 & 121 & $<0.0001$ & 233 & 131 & 212 & 113 & 218 & 124 & 212 & 110 & 0.0002 \\
\hline Protein (g/d) & 65 & 33 & 72 & 31 & 77 & 32 & 85 & 33 & $<0.0001$ & 74 & 36 & 73 & 33 & 77 & 33 & 77 & 32 & $<0.0001$ \\
\hline $\begin{array}{l}\text { Total energy } \\
\text { contribution from } \\
\text { protein }(\%)\end{array}$ & 20 & 5 & 20 & 4 & 20 & 4 & 21 & 4 & $<0.0001$ & 22 & 4 & 21 & 4 & 20 & 4 & 19 & 4 & $<0.0001$ \\
\hline Carbohydrate (g/d) & 145 & 73 & 161 & 68 & 172 & 73 & 188 & 76 & $<0.0001$ & 136 & 59 & 157 & 66 & 170 & 68 & 194 & 74 & $<0.0001$ \\
\hline $\begin{array}{l}\text { Total energy } \\
\text { contribution from } \\
\text { carbohydrate (\%) }\end{array}$ & 43 & 8 & 45 & 8 & 45 & 8 & 46 & 7 & $<0.0001$ & 41 & 7 & 44 & 7 & 45 & 7 & 48 & 7 & $<0.0001$ \\
\hline Sugar $(g / d)$ & 66 & 36 & 74 & 37 & 80 & 39 & 87 & 38 & $<0.0001$ & 64 & 32 & 71 & 35 & 79 & 36 & 90 & 40 & $<0.0001$ \\
\hline Fibre $(\mathrm{g} / \mathrm{d})$ & 15 & 8 & 18 & 8 & 20 & 9 & 23 & 10 & $<0.0001$ & 14 & 7 & 17 & 7 & 19 & 8 & 23 & 10 & $<0.0001$ \\
\hline Alcohol $(\mathrm{g} / \mathrm{d})$ & 2 & 12 & 4 & 15 & 6 & 16 & 6 & 16 & $<0.0001$ & 15 & 25 & 6 & 17 & 3 & 11 & 2 & 8 & $<0.0001$ \\
\hline $\mathrm{Na}(\mathrm{mg} / \mathrm{d})$ & 1749 & 872 & 1890 & 843 & 2007 & 915 & 2209 & 944 & $<0.0001$ & 1832 & 912 & 1887 & 931 & 1985 & 984 & 2078 & 969 & $<0.0001$ \\
\hline $\mathrm{K}(\mathrm{mg} / \mathrm{d})$ & 2127 & 956 & 2392 & 903 & 2580 & 958 & 2908 & 1040 & $<0.0001$ & 2195 & 930 & 2363 & 975 & 2559 & 979 & 2787 & 1056 & $<0.0001$ \\
\hline$M g(m g / d)$ & 203 & 94 & 239 & 98 & 260 & 105 & 297 & 120 & $<0.0001$ & 211 & 95 & 233 & 103 & 255 & 107 & 286 & 122 & $<0.0001$ \\
\hline$\beta$-Carotene $(\mu \mathrm{g} / \mathrm{d})$ & 2660 & 2331 & 3291 & 2245 & 3548 & 2409 & 4134 & 2482 & $<0.0001$ & 2597 & 2021 & 3043 & 2016 & 3552 & 2273 & 4268 & 2662 & $<0.0001$ \\
\hline Vitamin C & 73 & 57 & 96 & 68 & 110 & 72 & 131 & 72 & $<0.0001$ & 73 & 47 & 91 & 60 & 106 & 68 & 139 & 77 & $<0.0001$ \\
\hline
\end{tabular}

ARFS, Australian Recommended Food Score; MDS, Mediterranean Diet Score; Q, quartile; IQR, interquartile range.

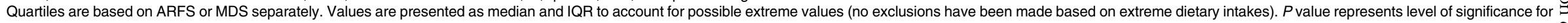
difference found through ANOVA for continuous variables. 
of vegetables (Q4, median $=2.3$ servings $/ \mathrm{d})$, fruits $(\mathrm{Q} 4$, median $=2.5$ servings $/ \mathrm{d}$ ) and grains $(\mathrm{Q} 4$, median $=4.2$ servings/d). On the other hand, higher ARFS was associated with higher average intakes of dairy (ARFS $\mathrm{Q} 4$, median $=1.9$ servings $/ \mathrm{d} v$. MDS $\mathrm{Q} 4$, median $=1.6$ servings/d), processed meat (ARFS Q4, median $=0.2$ servings/d $v$. MDS $\mathrm{Q} 4$, median $=0.1$ servings $/ \mathrm{d}$ ) and meat $($ ARFS Q4, median $=1.9$ servings $/ \mathrm{d} v$. MDS Q4, median $=1.6$ servings $/$ d) compared with higher MDS, and may contribute to the different effect sizes we observed between the two scores.

With these underlying differences between the MedDiet and ADG in mind, there have been concerns that the adoption of a traditionally low-dairy MedDiet would not be able to effectively meet the Ca recommendations for Western populations ${ }^{(34)}$. To address this notion, an 8-week randomised controlled trial aimed to determine the effect of the MedDiet supplemented with dairy foods on cardiovascular risk factors in participants ( $n$ 38) at risk of CVD $^{(34)}$. Results indicated that a MedDiet including 3-4 servings dairy/d led to significant reductions in blood pressure, higher HDL-cholesterol and lower TAG levels, compared with a low-fat diet ${ }^{(34)}$. In addition, other studies have indicated that dairy intake is associated with markers of cardiovascular health ${ }^{(35,36)}$. While a meta-analysis of prospective cohort studies concluded dairy consumption may be associated with a reduced risk of CVD, additional data are required to examine potential dose-response patterns $^{(37)}$.

Olive oil is a major component of the MedDiet. Although the Spanish cohort of the European Prospective Investigation into Cancer and Nutrition (EPIC-Spain) indicated that the majority of the MedDiet components on their own were not significantly associated with mortality (rather the sum of its components is important), higher intake of olive oil was associated with a significant $15 \%$ lower risk of mortality $(P=0.006)^{(38)}$. In our cohort, better adherence to the ARFS and the MDS was associated with a lower average percentage of total energy from fat and higher average intakes $(\mathrm{g} / \mathrm{d})$ of monounsaturated fat and polyunsaturated fat. Saturated fat intake was on average significantly lower with increasing adherence to the MDS; however, average saturated fat intake did not change significantly based on adherence to the ARFS. It is worth noting here that the ADG do not recommend a specific type of unsaturated fat (polyunsaturated or monounsaturated) and in only small amounts, given that essential unsaturated fatty acids are found in a variety of core foods ${ }^{(15)}$. However, this also represents a potential limitation of the current analysis. Given olive oil is a major component of the MedDiet, our current analysis was not able to accurately account for this as it was not collected as part of the DQES. Alternatively, the MDS fat component score was awarded if women reported consuming monounsaturated fat spreads (major type of fat in olive oil) ${ }^{(39)}$. As a result, the 
true effect of the MDS on hypertension and non-fatal CVD in our cohort may be underestimated.

Australians are considered to be one of the highest global consumers of meat ${ }^{(40)}$. We found average meat and processed meat intakes to be higher in women consuming diets more aligned with the ADG compared with the MDS, and this could help explain why we observed slightly greater risk reductions with increasing adherence to the MDS. In fact, previous prospective cohort analyses have indicated that population adherence to dietary patterns with higher red meat intake is significantly associated with markers of systemic inflammation, including C-reactive protein ${ }^{(41,42)}$. Additionally, recent data indicate that people who consume more red meat produce higher levels of trimethylamine $\mathrm{N}$-oxide, a microbiome-mediated metabolite that has been found to have a role in the pathogenesis of atherosclerotic heart disease ${ }^{(43)}$. However, trimethylamine $N$-oxide levels were markedly reduced when study participants stopped consuming red meat in favour of either white meat or a vegetarian diet $^{(43)}$. In addition, trimethylamine $N$-oxide levels have been shown to be especially low in those following a MedDiet, compared with individuals consuming a Western diet ${ }^{(44)}$.

In our cohort we found women consuming diets more aligned with the MDS were also more likely to consume discretionary choices (inclusive of confectionery, salty snacks, takeaway and pastries). Higher intakes of commercial sweets and salty snacks are not consistent with the MedDiet, but likely a reflection of modern-day food environments ${ }^{(45)}$. This may be unexpected given that with increased multiculturalism in modern Australia, improved technology and globalisation there has been an increase in the availability of several MedDiet components including fruits, vegetables and olive oil ${ }^{(45)}$. However, the availability of highly processed discretionary choices in Australia has increased at an alarmingly rapid rate compared with traditional Mediterranean foods, thus impacting the balance of available foods and affecting adherence with the MedDiet, a concept which can also be extended to explain low adherence to the $\mathrm{ADG}^{(45)}$.

It has been previously indicated that a MDS of at least half of the maximum possible points represents satisfactory adherence to the MedDiet and is associated with better health outcomes ${ }^{(33)}$. Applying this logic to our current cohort, only women in the highest quartile for the MDS had satisfactory compliance (median score of 8 out of a possible 17). A similar level of compliance was also noted for the ARFS (median score of 41 out of a possible 74), further reinforcing the notion that there is still much room for improving the diets of Australian women. However, it was encouraging to observe in this cohort that satisfactory adherence (i.e. Q4 scores) to both diet quality scores were associated with moderate-to-large statistically significant risk reductions in hypertension and non-fatal CVD. Further, although our analysis was limited due to insufficient power to analyse all specific non-fatal CVD types 
separately, our findings indicate that diet can influence the development of CVD risk factors differently and remains a factor worth further exploration.

Analyses of whole diets through the use of diet quality scores is a major strength of the current analysis. This is due to their ability to summarise the complexity of diets, taking into account the synergistic effects or interactions between nutrients and foods which make up whole diets, a concept which is ignored with the analysis of single nutrients/food groups $^{(33)}$. It is important to mention that higher ARFS, indicating better compliance with ADG, was associated with a significantly reduced odds of non-fatal CVD and hypertension. However, we observed a markedly lower odds of hypertension, and a statistically significant overall trend for lower non-fatal CVD, with greater compliance to the MDS. Interestingly, most scientific knowledge used as evidence for the creation of current national dietary guidelines is based on associations between foods or nutrients and incidence of certain diseases. These studies have been vital for building our baseline scientific knowledge within this field, and prospective cohort analyses currently represent the best methodology for understanding the long-term impacts of dietary components in free-living participants. However, given diet quality scores have been consistently shown to be better predictors of health than any individual dietary component ${ }^{(38)}$, the next review of the evidence informing the ADG should also consider building the body of evidence around diet quality and long-term health.

For the current investigation, our exposure (diet), outcomes (hypertension and non-fatal CVD) and covariates (i.e. BMI and physical activity) relied on self-reported data, thus representing a limitation of our study, due to the inherent errors associated with self-reported data. It is worth noting, however, that much of these data has been previous validated in this cohort, including many of the covariates, the use of the DQES and the use of self-reported outcomes including hypertension and stoke. Further, the data presented in the current analysis are in line with the expected number of non-fatal CVD in female Australian women, based on other national estimates ${ }^{(46-48)}$. However, it should also be noted that despite the use of a directed acyclic graph to inform covariate selection for the multivariate analysis, residual confounding is possible and could be distorting our findings.

\section{Conclusion}

Better diet quality scores were associated with a lower risk of hypertension and non-fatal CVD within a sample of middle-aged Australian women followed-up for over 15 years. In particular, we observed greater risk reduction in hypertension with increasing adherence to the MDS compared with the ARFS. These findings add to the evidence base indicating that within an Australian context, better adherence to the MedDiet is associated with a lower risk of hypertension, but not necessarily non-fatal CVD. Further, these findings indicate the need for updated evidenced-based ADG and public health nutrition policies. However, continued epidemiological analyses within Australian cohorts are required to further develop an understanding of whether MedDiet-inspired ADG will be a useful strategy to protect and preserve the health of the population.

\section{Acknowledgements}

Acknowledgements: The research on which this paper is based was conducted as part of the Australian Longitudinal Study on Women's Health by the University of Newcastle and University of Queensland. The authors are grateful to the Australian Government Department of Health for funding and to the women who provided the survey data. The authors thank Professor Graham Giles, from the Epidemiological Centre of the Cancer Council Victoria, for permission to use the Dietary Questionnaire for Epidemiological Studies (Version 2), 1996. Financial support: This research received no specific grant from any funding agency in the public, commercial or not-for-profit sectors. Conflict of interest: None. Authorship: J.K.J., A.J.P., L.K.M.-W. and M.A.M. were responsible for formulating the research question. J.K.J., P.M.F., C.H., C.O., J.E.B. and M.A.M. were responsible for study design and formulation of the analysis plan. J.K.J. was responsible for carrying out the analysis and primarily responsible for drafting the manuscript. All authors contributed to and reviewed the final version of the manuscript. Ethics of human subject participation: This study was conducted according to the guidelines laid down in the Declaration of Helsinki and all procedures involving human subjects were approved by the University of Newcastle (approval number H-076-0795) and the University of Queensland (approval number 200400224). Written informed consent was implied by all participants at the return of a completed survey.

\section{Supplementary material}

To view supplementary material for this article, please visit https://doi.org/10.1017/S1368980019002842

\section{References}

1. American Heart Association (2017) What is cardiovascular disease? http://www.heart.org/en/health-topics/consumer-health care/what-is-cardiovascular-disease (accessed September 2018).

2. Laslett LJ, Alagona P, Clark BA et al. (2012) The worldwide environment of cardiovascular disease: prevalence, diagnosis, therapy, and policy issues: a report from the American College of Cardiology. J Am Coll Cardiol 60, 25 Suppl., S1-S49. 
3. Australian Institute of Health and Welfare (2010) Women and Heart Disease: Summary. Cardiovascular Disease Series no. 34. Catalogue no. CVD 50. Canberra, ACT: AIHW.

4. World Health Organization, World Heart Federation \& World Stroke Organization (2011) Global Atlas on Cardiovascular Disease Prevention and Control. Geneva: WHO.

5. Maticevic J, Miller L, Girschik J et al. (2017) Projecting Preventable Cardiovascular Disease Burden Due to Modifiable Risk Factors in Western Australia in 2025. Perth, WA: Heart Foundation.

6. Tapsell LC, Neale EP, Satija A et al. (2016) Foods, nutrients, and dietary patterns: interconnections and implications for dietary guidelines. Adv Nutr 7, 445-454.

7. Widmer RJ, Flammer AJ, Lerman LO et al. (2015) The Mediterranean diet, its components, and cardiovascular disease. Am J Med 128, 229-238.

8. Stefler D, Malyutina S, Kubinova R et al. (2017) Mediterranean diet score and total and cardiovascular mortality in Eastern Europe: the HAPIEE study. Eur J Nutr 56, 421-429.

9. Nissensohn M, Román-Viñas B, Sánchez-Villegas A et al. (2016) The effect of the Mediterranean diet on hypertension: a systematic review and meta-analysis. J Nutr Educ Behav $\mathbf{4 8}$, 42-53.e1.

10. Nordmann AJ, Suter-Zimmermann K, Bucher HC et al. (2011) Meta-analysis comparing Mediterranean to low-fat diets for modification of cardiovascular risk factors. Am J Med 124, 841-851.e2.

11. Grosso G, Marventano S, Yang J et al. (2017) A comprehensive meta-analysis on evidence of Mediterranean diet and cardiovascular disease: are individual components equal? Crit Rev Food Sci Nutr 57, 3218-3232.

12. Dernini S \& Berry EM (2015) Mediterranean diet: from a healthy diet to a sustainable dietary pattern. Front Nutr $\mathbf{2}, 15$.

13. Trichopoulou A \& Lagiou P (1997) Healthy traditional Mediterranean diet: an expression of culture, history, and lifestyle. Nutr Rev 55, 383-389.

14. US Department of Agriculture (2018) Dietary guidelines from around the world. https://www.nal.usda.gov/fnic/dietaryguidelines-around-world (accessed December 2018).

15. National Health and Medical Research Council (2013) Australian Dietary Guidelines. Canberra, ACT: NHMRC.

16. Jackson J, Patterson A, MacDonald-Wicks L et al. (2018) Dietary nitrate and diet quality: an examination of changing dietary intakes within a representative sample of Australian women. Nutrients 10, 1005.

17. Brown WJ, Bryson L, Byles JE et al. (1999) Women's Health Australia: recruitment for a national longitudinal cohort study. Women Health 28, 23-40.

18. Brown WJ, Young AF \& Byles JE (1999) Tyranny of distance? The health of mid-age women living in five geographical areas of Australia. Aust J Rural Health 7, 148-154.

19. Lee C, Dobson AJ, Brown WJ et al. (2005) Cohort profile: the Australian Longitudinal Study on Women's Health. Int J Epidemiol 34, 987-991.

20. Giles G \& Ireland P (1996) Dietary Questionnaire for Epidemiological Studies (Version 2). Melbourne, VIC: Cancer Council Victoria.

21. Hodge A, Patterson AJ, Brown WJ et al. (2000) The Anti Cancer Council of Victoria FFQ: relative validity of nutrient intakes compared with weighed food records in young to middle-aged women in a study of iron supplementation. Aust N Z J Public bealth 24, 576-583.

22. Collins CE, Young AF \& Hodge A (2008) Diet quality is associated with higher nutrient intake and self-rated health in mid-aged women. J Am Coll Nutr 27, 146-157.

23. Vissers L, Waller M, van der Schouw Y et al. (2017) A proinflammatory diet is associated with increased risk of developing hypertension among middle-aged women. Nutr Metab Cardiovasc Dis 27, 564-570.
24. Jackson CA, Mishra GD, Tooth L et al. (2015) Moderate agreement between self-reported stroke and hospital-recorded stroke in two cohorts of Australian women: a validation study. BMC Med Res Methodol 15, 7.

25. Jackson CA, Dobson AJ, Tooth LR et al. (2016) Lifestyle and socioeconomic determinants of multimorbidity patterns among mid-aged women: a longitudinal study. PLoS One 11, e0156804.

26. World Health Organization (2006) Global Database on Body Mass Index: BMI classification. http://www. assessmentpsychology.com/icbmi.htm (accessed October 2018).

27. Burton NW, Brown W \& Dobson A (2010) Accuracy of body mass index estimated from self-reported height and weight in mid-aged Australian women. Aust N Z J Public Health 34, 620-623.

28. Armstrong T, Bauman AE \& Davies J (2000) Physical Activity Patterns of Australian Adults: Results of the 1999 National Physical Activity Survey. Canberra, ACT: Australian Institute of Health and Welfare.

29. National Health and Medical Research Council (2009) Australian Guidelines to Reduce Health Risks from Drinking Alcohol. Canberra, ACT: NHMRC.

30. Berecki-Gisolf J, Begum N, \& Dobson AJ (2009) Symptoms reported by women in midlife: menopausal transition or aging? Menopause 16, 1021-1029.

31. Textor J, Hardt J \& Knüppel S (2011) DAGitty: a graphical tool for analyzing causal diagrams. Epidemiology 22, 745.

32. Sofi F, Abbate R, Gensini GF et al. (2010) Accruing evidence on benefits of adherence to the Mediterranean diet on health: an updated systematic review and meta-analysis. Am J Clin Nutr 92, 1189-1196.

33. Bach A, Serra-Majem L, Carrasco JL et al. (2006) The use of indexes evaluating the adherence to the Mediterranean diet in epidemiological studies: a review. Public Health Nutr 9 , 132-146.

34. Wade AT, Davis CR, Dyer KA et al. (2018) A Mediterranean diet supplemented with dairy foods improves markers of cardiovascular risk: results from the MedDairy randomized controlled trial. Am J Clin Nutr 108, 1166-1182.

35. Crichton GE, Elias MF, Dore GA et al. (2012) Relations between dairy food intake and arterial stiffness: pulse wave velocity and pulse pressure. Hypertension 59, 1044-1051

36. Drouin-Chartier J-P, Brassard D, Tessier-Grenier $\mathrm{M}$ et al. (2016) Systematic review of the association between dairy product consumption and risk of cardiovascular-related clinical outcomes. Adv Nutr 7, 1026-1040.

37. Alexander DD, Bylsma LC, Vargas AJ et al. (2016) Dairy consumption and CVD: a systematic review and meta-analysis. BrJ Nutr 115, 737-750.

38. Buckland G, Agudo A, Travier N et al. (2011) Adherence to the Mediterranean diet reduces mortality in the Spanish cohort of the European Prospective Investigation into Cancer and Nutrition (EPIC-Spain). Br J Nutr 106, $1581-1591$.

39. Martínez-González MÁ \& Sánchez-Villegas A (2004) The emerging role of Mediterranean diets in cardiovascular epidemiology: monounsaturated fats, olive oil, red wine or the whole pattern? Eur J Epidemiol 19, 9-13.

40. Organisation for Economic Co-operation and Development (2018) Meat consumption. https://data.oecd.org/agroutput/ meat-consumption.htm (accessed December 2018).

41. Damiao R, Castro TG, Cardoso MA et al. (2006) Dietary intakes associated with metabolic syndrome in a cohort of Japanese ancestry. BrJ Nutr 96, 532-538.

42. Esmaillzadeh A, Kimiagar M, Mehrabi Y et al. (2007) Dietary patterns and markers of systemic inflammation among Iranian women. J Nutr 137, 992-998. 
43. Wang Z, Bergeron N, Levison BS et al. (2018) Impact of chronic dietary red meat, white meat, or non-meat protein on trimethylamine $N$-oxide metabolism and renal excretion in healthy men and women. Eur Heart J 40, 583-594.

44. De Filippis F, Pellegrini N, Vannini L et al. (2016) High-level adherence to a Mediterranean diet beneficially impacts the gut microbiota and associated metabolome. Gut $\mathbf{6 5}$, 1812-1821.

45. da Silva R, Bach-Faig A, Quintana BR et al. (2009) Worldwide variation of adherence to the Mediterranean diet, in 19611965 and 2000-2003. Public Health Nutr 12, 1676-1684.
46. Australian Bureau of Statistics (2015) National Health Survey: first results, 2014-15. http://www.abs.gov.au/ausstats/abs@. nsf/Lookup/by\%20Subject/4364.0.55.001 2014-15 Main\% 20Features Hypertension\%20and\%20measured\%20high\% 20blood\%20pressure 14 (accessed November 2018).

47. Catanzariti L \& Senes S (2010) Women and Heart Disease: Cardiovascular Profile of Women in Australia. Canberra, ACT: Australian Institute of Health and Welfare.

48. Ageno W, Squizzato A, Garcia D et al. (2006) Epidemiology and risk factors of venous thromboembolism. Semin Thromb Hemost 32, 651-658. 